\title{
Qualitative Systematic Literature Review: The Experience of Being in Seclusion for Adults with Mental Health Difficulties
}

\section{Abstract}

Purpose: To conduct a systematic search of the peer-reviewed qualitative literature investigating the lived experience of seclusion for adults with mental health difficulties, to appraise the quality of the existing literature and synthesise findings. Background:Seclusion is a controversial intervention for the short-term management of unsafe behaviours in inpatient mental health services. There has been some sporadic interest in the service-users' experiences of this. Design: Systematic literature review and meta-synthesis. Data Sources: Databases MEDLINE, EMBASE, CINAHL and PSYCHINFO were searched in July 2015. Review Methods: The JBI QARI tools for critical appraisal and data extraction were used to review papers and synthesise findings. Findings: A small number of papers was found, which were of mixed quality. Value: The existing research is limited in both quantity and quality. Although most participants from the existing research described seclusion as mostly negative with the potential for causing iatrogenic harm, some described more positive experiences, often in the context of compassionate interactions with staff.

\section{Summary Statement:}

\section{Why is this research or review needed?}

- The use of seclusion is common within mental health services but there is an absence of evidence for the purported theoretical rationale for its use.

- Studies of the experiences of individuals placed in seclusion are small in number, of mixed quality and with mixed findings. 
- A systematic literature review and meta-synthesis of the existing qualitative literature investigating the lived experience of seclusion for adults with mental health difficulties was conducted, to synthesize the existing evidence base and make suggestions for future developments in research and practice.

\section{What are the key findings?}

- The existing research is limited both in quantity and quality.

- Despite the limitations of existing research, the evidence does not support the purported theoretical rationale for the therapeutic use of seclusion. This poses a significant challenge to a common practice within mental health settings.

- Seclusion has the potential to cause iatrogenic harm, particularly where interactions with nursing staff are not experienced as compassionate.

- The actions of nursing staff in implementing seclusion procedures may mitigate iatrogenic harm.

- Individuals who have experienced seclusion have suggestions for how to improve its use.

\section{How should the findings be used to influence policy / practice / research / education?}

- There is clearly scope for further, high quality research into people's experiences of seclusion, particularly within the UK.

- The findings include concrete ways in which the practice of seclusion can be improved.

- Action research methods may offer a useful way of implementing and evaluating changes in practice. 


\section{$\underline{\text { Key Words }}$}

Qualitative, Systematic Reviews and Meta-analyses, Mental Health, Acute Care, Quality of Care, Psychiatric Nursing

\section{$\underline{\text { Introduction }}$}

Seclusion, also known as supervised-confinement, is one of the oldest interventions still in use for those with mental health difficulties, but is considered controversial by many (McCoy and Garritson, 1983). Seclusion practices vary internationally and there is no clear, consistent definition (Happell and Harrow, 2010). Common amongst these practices is the placement of the individual alone in a locked room for their own protection and that of their environment, with the aim of controlling unsafe and aggressive behaviours and allowing nursing and treatment interventions (Lendemeijer and Shortridge-Baggett, 1997; Sailas and Fenton, 2012; Happell and Harrow, 2010). Despite controversies and policies stipulating it be used only as a last resort, seclusion continues to be a commonly used intervention on inpatient mental health units. The 2010 'Count Me In' national census of mental health and learning disability service inpatients in England and Wales (Care Quality Commission, 2010) found that four per cent of adult mental health service users had experienced one or more episodes of seclusion. This was similar to five previous similar censuses. However, in all six censuses there were significant differences between ethnic groups, with White British individuals having a seclusion rate that was nine per cent lower than average in the 2010 census (Care Quality Commission [CQC], 2010).

\section{Background}


The proposed rationale for seclusion were that it provides three important elements to help the individual feel safe from external stimuli that may have led to disruptive behaviour, namely containment, isolation and a reduction in sensory stimulation (Gutheil, 1978). This is based on the assumptions that increased sensitivity to sensory stimulation may be present in psychosis, and that those with a heightened sensitivity to environmental stimuli may be unable to tolerate competing sensory demands of an inpatient mental health unit (Wells, 1972; Rosen and DiGiacomo, 1978). However, these theoretical underpinnings of seclusion have received little investigative attention since and there has been little development to better understand this (Alty and Mason 1994). In addition, it has been questioned whether the use of seclusion is a therapeutic intervention, a form of emergency containment or punishment (Mason, 1993). Parallels between isolation imposed for therapeutic reasons during seclusion and the isolation imposed as punishment in solitary confinement (Farrell and Dares, 1996) and research exploring experiences within inpatient mental health settings have found seclusion to be harmful for many (Frueh et al., 2005).

It is recognised that seclusion risks infringing the rights of service users and may be viewed by them as punitive rather than calming or protective (Mayers et al., 2010). This may particularly be the case when the prospect of seclusion is used as a 'threat', as in an example given by a service user of being 'threatened with seclusion just for kicking the ward door once in a moment's temper because I couldn't get hold of my care coordinator on the phone' (Anoniou, 2007, p. 125). Seclusion may also be seen as punitive if alternative measures are not attempted or used during the process. One study found that there were no 'therapeutic' nursing interventions documented during $69 \%$ of seclusion events $(\mathrm{N}=29)$ during the study (O'Brien and Cole, 2004). However, medical and nursing staff can identify and use alternatives to minimise the use of seclusion (Konito et al., 2010). There have been some 
examples of organisational initiatives that have led to significant reductions in the use of seclusion through the introduction of alternative strategies (e.g. Taxis, 2009).

Guidance and protocols for the use of seclusion vary within the UK and are outlined by specific NHS or other hospital trusts (NICE, 2015; NICE, 2005). However, UK national guidance stipulates seclusion must only be used: on individuals detained under the Mental Health Act; as a last resort; in accordance with core procedural standards; and that the dignity of the individual must be upheld (NICE 2015). Despite existing guidelines in the UK, the Care Quality Commission (2015) report that inspections has found some hospital seclusion facilities to be unfit for purpose and gives a range of examples of poor practice, including inadequate furnishing, little or no access to toilets; and disturbances by staff playing the radio or talking loudly in the observation area. It is recognised that there may be differences in the use of seclusion internationally. However, internationally there are ethical and moral dilemmas in using seclusion, which can contributed to conflicting and distressing emotions for nursing staff implementing such practice (Khalifeh, 2015).

Research investigating the effectiveness of seclusion is contradictory. Some reviews have concluded that seclusion is an effective intervention in preventing violence and self-harm (Fisher 1994; Lendemeijer and Shortridge-Baggett, 1997) but another concluded that there is no good quality evidence in support of this (Sailas and Fenton, 2012). This is likely due to the impossibility of conducting randomised controlled trials ethically for such phenomenon. Another systematic review of qualitative and quantitative research concluded that due to the poor quality of existing literature, there is insufficient evidence on the effectiveness or safety of seclusion as a short-term intervention for the management of violence in adult inpatient mental health units (Nelstrop et al., 2006). Of note, seclusion has been found to have the potential to increase rather than reduce aggressive behaviour (Donat, 2003). Interestingly, 
the reduction in use and elimination of seclusion has been found not to lead to an increase in staff injury (Ashcraft and Anthony, 2008; Martin et al., 2008). Furthermore, a smaller recovery-oriented crisis service found elimination of seclusion resulted in a decrease of yearly staff injuries from fifteen to five (Ashcraft and Anthony, 2008).

It is vital to understand how such a controversial intervention is experienced by those involved. Some studies have found that nurses are distressed by and dissatisfied with restrictive interventions such as seclusion (Moran et al., 2009; Duxbury and Whittington, 2005). A review of the literature has concluded that most nurses view seclusion as a necessary intervention in the management of violence and aggression (Happell and Harrow, 2010), and refer to strict protocols for the use of seclusion to appease ethical concerns (MuirCochrane, 1996). Interest in service users' experiences of seclusion has been sporadic. There are some examples of innovative approaches to research in this field, such as participatory studies involving service users throughout the process (Mayers et al., 2010). However, there has been no synthesis of research into service users' perspectives of seclusion. There have long been drives to reduce and even eliminate the use of seclusion, with varying success rates (Donat, 2003; Geller, 2007). Therefore, it was considered timely to synthesise the published peer-reviewed research on the lived experience of seclusion for adults with mental health difficulties, to further inform public policy and future research on the experience of seclusion.

\section{The Review}

\section{$\underline{\text { Aims }}$}

There were three aims of this review: i) to conduct a systematic search of the existing peerreviewed qualitative research investigating the lived experience of seclusion for adults with 
mental health difficulties. ii) To critically appraise the quality of the existing literature. iii) To produce a synthesis of research findings.

\section{$\underline{\text { Design }}$}

The systematic literature review and meta-synthesis of findings was conducted following the protocol outlined by the Joanna Briggs Institute (JBI: The Joanna Briggs Institute, 2009).

\section{Search Method}

Four academic and research databases were searched in July 2015: Medline, EMBASE, Psychinfo and CINAHL. The following search terms were used: service user ${ }^{\star}$, patient ${ }^{\star}$, or client $^{*}$ and mental health, psychiatric or inpatient and experience* ${ }^{*}$ perception ${ }^{\star}$, perceive, attitude, impact ${ }^{*}$, describe* ${ }^{*}$ description*, opinion* or feel ${ }^{\star}$ about and seclusion, patient seclusion, seclusion room, supervised confinement, confined, coercion, coercive intervention*, restrictive intervention*, physical restrain, restricted, segregat ${ }^{*}$, confinement or contain*. In addition, further studies were sought by hand searching the references in all included studies and literature reviews found.

\section{$\underline{\text { Inclusion Criteria }}$}

Qualitative research from peer-review journals was included if it: i) focused on adult serviceusers' experience of seclusion ii) presented original data iii) included participants with firsthand experience of being placed in seclusion.

\section{Exclusion Criteria}

Papers were excluded if: i) they were not written in the English language ii) it was not possible to disaggregate data of adults with mental health difficulties' first hand experiences 
of being in seclusion iii) qualitative data (such as quotations from interviews) could not be extracted.

\section{Search Outcome}

The search outcome and included studies are illustrated in the QUOROM Diagram (figure 1.) and Table 1. Each included study was assigned a number $(1-11)$, which is used to refer to the papers throughout.

Figure 1 approximately here

Fig. 1. QUOROM Diagram Illustrating the Selection

Table 1 approximately here:

Table 1. Table of included studies Key findings are presented as the themes presented in each paper.

\section{Quality Appraisal}

The JBI's Qualitative Assessment and Review Instrument (QARI) was used to systematically appraise the quality of included studies; it forms part of the JBI manual for systematic reviews and is advocated by the Journal of Advanced Nursing. However, a critique of this tool is that criteria are rated 'yes' or 'no', meaning the degree to which a criterion is met cannot be accounted for. Many of the papers included in this study met criterion to varying levels. Further, a number of papers discussed ethical issues without stating obtainment of ethical 
approval but this cannot be accounted for if the QARI tool is followed strictly. Finally, the the QARI tool does not account for potential bias in participant selection, for example some papers stated that ward staff identified 'appropriate' participants.

The papers identified were characterised as a series of small-scale qualitative studies of mixed quality. All of the papers demonstrated congruity between the research methodology, research questions, data analysis and collection and interpretation of the results. However, the majority of papers did not locate the researchers culturally or theoretically, or address the influence of the researcher. Papers which met fewer of the quality appraisal criteria, such as Richardson (1987) and Larue et al. (2013) tended to use fewer quotations to illustrate their findings. This arguably means that their participants' voices were less adequately represented than papers that used more illustrative quotes to support themes. Table 2 below illustrates the assessed quality of each of the included studies.

Table 2 approximately here.

\section{Table 2. Table to Illustrate Quality Appraisal}

\section{Data Abstraction}

Data were extracted from all 11 papers using the QARI tool, by both researchers independently. Where there was disagreement, this was resolved through discussion. Where possible themes were extracted as described by the authors with illustrative quotations from their participants. The JBI QARI data extraction tool restricts the extraction of data to clearly defined themes when used strictly. It was used flexibly in this synthesis to allow for the extraction of poorly defined themes.

\section{Synthesis}


The inductive approach to synthesise the extracted data is illustrated in Table 3. Themes were identified and extracted from the included papers and merged into one where there was significant overlap in themes from different papers. A total of 27 themes were identified. An inductive approach was then used to synthesise these: they were initially grouped together based on similarity, forming eleven subcategories. Subcategories were then reviewed and grouped together where they described a common process or experience, forming five categories and leading to an overall synthesis of the literature. The five categories identified were i) Emotional Impact of Seclusion, ii) Environmental Experience of Seclusion, iii) Cognitive and Behavioural Responses to Being in Seclusion, iv) Making Sense of the Seclusion Experience and v) Role of staff. These and the overall synthesis of the literature are explored below. Please note that for brevity, papers are referred to by the number assigned to them in Table 1.

Table 3 approximately here.

Table 3. Table to Illustrate the Inductive Synthesis Process

The Emotional Impact of Seclusion

Ten papers (all except paper 7) identified the emotional impact of seclusion as an important theme. All of these found that participants reported negative emotional experiences in seclusion. Two papers $(4,10)$ also found that some participants reported positive effects of the seclusion experience.

i) Negative Emotional Response

All papers reviewed (other than paper 7), identified themes of negative emotional responses to seclusion. These themes had titles such as intense affect, emotional impact, emotional experiences, loneliness, autonomy, patient emotional response to the seclusion process and 
feelings. In common, participants from the included papers described feelings of fear, anger, frustration, powerlessness and sadness. For example:

...I was feeling very low, I couldn't have felt any lower I thought, until they put me in seclusion and then I realized you could go lower. But by then there was nothing I could do about it. They even take away your option to try and change your circumstances to try and lift your mood (Meehan et al. 2000 p. 373).

...I felt fear and anger, especially towards those who put me into the seclusion room. Nurses and physicians used power and authority over patients. I didn't know where I was and how long it lasted, it was terrible.... (Kontio et al. 2012 p. 19).

One study identified that the experience of loneliness could extended to after seclusion had ended, due to a lack of understanding from others about the experience:

I've talked about it with my dad and with my sister too. Somehow I feel it may even be incredible, in a way. As if what you feel and what you experience at such a time that this to other people is... that other people cannot fully live this experience (Hoekstra et al. 2004 p.280).

Similarly, Hoekstra et al. (2004) identified that seclusion impacted on participants' trust and self-confidence both at the time of seclusion and for some time afterwards, which impacted on their relationships and ability to openly share thoughts, feelings and emotions with even relatives and close friends.

Although Sambrano and Cox (2013) (7) did not describe a theme of negative emotional responses, the anger participants felt when recounting their experiences comes across in quotations, e.g. 
...They would just take me to seclusion and just give me the injection! And they just

leave me for the night let me out in the morning. You know? And when l'm there, and, Ahhh!! [Peter yells in anger] (Sambrano and Cox 2013 p.525).

\section{ii) Positive Emotions}

Two of the papers $(4,10)$ also described positive emotions in relation to the seclusion experience, under the theme of 'positive effects'. For example, one participant commented on feeling 'relieved I'm in good secure hands so I can get some sleep' (ElBadri and Mellsop, 2008, p. 251). It is important to note that El-Badri and Mellsop (2008) used a questionnaire with closed questions and a space for qualitative comments. The ten closed questions in the questionnaire are not described in the paper. Further, Richardson (1987) gives little evidence from the data to support her findings.

\section{The Environmental Experience of Seclusion}

Four papers $(1,2,3,8)$ reported on the environmental experiences of seclusion, either the physical experience of the seclusion room itself or the events of the seclusion process. Other papers also described these aspects of the participants' experiences of seclusion under different themes.

\section{i) The Seclusion Room Environment}

The seclusion room environment was explored either within themes such as 'sensory deprivation' (2), descriptions of problems relating to basic needs (3), suggestions for improvements $(3,8)$ and 'objects' $(8)$, which related to items participants came into contact with in seclusion. Participants from studies described the sense of being '...locked up with all my problems and bewilderment' (Wadeson and Carpenter 1976 p. 322). Most striking were consistent reports about the lack of access to facilities to meet basic needs: 
...I kicked the door a long time so that they could understand my need to get to the toilet. Once I relieved myself on the porridge plate and put two sandwiches on it to prevent the smell...' (Kontio et al. 2012 p. 19).

...I was dirty, I sweated all the time. They washed my hair once a week and I didn't have a chance to brush my teeth. I was thirsty and I peed into the floor-drain... (Kontio et al. 2012 p. 19).

ii) The Events of Seclusion

One paper (8) described how participants reported that the physical events of seclusion, such as disrobing and the locking of the door were experienced to be 'frightening', 'humiliating' and 'dehumanizing' (Norris and Kennedy 1992 p. 10), this is echoed by other papers under different themes, such as Emotional Impact:

It's humiliating, having male staff seeing me naked and you've got to face them... Yeah, there was females ere too, but they don't care if there's male staff watching while you're naked, couldn't care less (Meehan et al. 2000 p. 373).

\section{The Cognitive and Behavioural Responses to Being in Seclusion}

Seven papers $(1,2,5,7,8,9,10,11)$ discussed participants' cognitive and behavioural responses to seclusion, particularly in relation to the impact on psychological symptoms and how they coped with seclusion.

i) Effects of Seclusion on Psychological Symptoms

Four papers $(1,2,8,11)$ explored the effects of seclusion on psychological symptoms, such as agitation, hallucinations, delusions and the effects of sensory deprivation. These symptoms may be perceived by staff as signs of illness or contributing to the need for 
seclusion. However, participants described these symptoms as either a response to being placed in seclusion, or as symptoms they already had which within seclusion were amplified rather than ameliorated. Such experiences, other than hallucinations, were consistently distressing for the participants involved:

I was locked in a gas chamber - behind bars. I was afraid of punishment but I don't remember for what. The room looked the same, but it was a gas chamber. I was waiting for the gas. There were strange odors and heat... Other patients had written obscenities on the wall. They were there for me - that's what my friends thought of me (Wadeson and Carpenter 1976 p. 322).

...the silence starts to drive you mad except for that blowing sound [fan in the ceiling] so you start talking to yourself, trying to keep yourself, you know, sane (Meehan et al. 2000 p. 374).

In contrast, Wadeson and Carpenter (1976) described how positive experiences of hallucinations in seclusion outweighed the negatives. Positive hallucinations were described as uplifting and reassuring; there is a possibility that these were a coping mechanism for those participants:

He [Mao Tse Tung] told me in Chinese to keep peace in the world. It was a surprising psychic communication... I felt important (Wadeson and Carpenter 1976 p. 320).

\section{ii) Coping}

Four papers $(5,7,9,10)$ illustrated how participants coped with their experiences in seclusion. For example, through comforting hallucinations, but also by maintaining control by talking to themselves, singing and resistance. Meehan et al. (2000) described submission as a form of coping: 
I just paced around, sung to myself, talked to myself did all these stupid little things that you do when you've got nothing else to do and you can't go no where else (Meehan et al. 2000 p. 374).

I just became so distressed that I didn't speak and stopped talking and just stopped moving and just thought maybe if I just keep still enough they'd come in and let me go out I didn't dare talk to anyone or do anything, you know cause I was frightened l'd go back in (Meehan et al. 2000 p. 374).

\section{Making Sense of the Seclusion Experience}

All included papers identified the ways in which participants made sense of their seclusion experience. For many, it was understood as a form of punishment and described as a dehumanising experience. Also identified were participants' reflections on their understanding of seclusion, or lack thereof. Participants were able to reflect on their experiences and suggest improvements to the use of seclusion.

i) Perception of Being Punished

Four of the included papers $(2,4,7,11)$ described themes where participants perceived seclusion as punishment or made sense of the experience as coercive and punishing. This was described under themes of the experience of being punished, the involvement of police in the seclusion process and the criminalisation of clients and the use of force. Feelings of punishment are also described as a part of other themes, such as delusions in seclusion and the emotional impact of the seclusion experience:

Or something just to show, don't mess with them. Don't mess with their standards and all that kind of stuff coz you get locked up (Sambrano and Cox 2013 p. 525). 
One participant described feeling like a 'prisoner without having done anything criminal' (ElBadri and Mellsop 2008 p. 250).

ii) Dehumanising Effects of Seclusion

Eight of the included papers $(2,3,4,5,6,7,9,11)$ described how participants made sense of their seclusion experience as dehumanising and humiliating. This came under themes of punishment, autonomy and problems relating to basic needs and was echoed in themes describing the emotional experience of seclusion and a sense of a loss of dignity and basic human rights:

...terrified not being told why I had been locked in this dark room on a mattress on the floor. I felt like a caged animal... (El-Badri and Mellsop 2008 p. 250).

I don't ever have a problem urinating myself, never. I could use the bathroom just fine, I can talk just fine, I can walk just fine. But to urinate myself and do that just because I was not given the chance to go to the bathroom. They refused to give me a pillow. They refused everything. All my rights were gone (Faschingbauer et al. 2013 p. 36).

iii) Understanding of Seclusion

Two papers $(6,8)$ illustrated themes of participants not being informed of or understanding the reasons for their placement in seclusion. A lack of prior knowledge of the use and process of seclusion was also identified:

...I didn't understand why they put me into the seclusion room and I never got information on this. The staff was reluctant to provide information on why, and how long, what next... (Kontio et al. 2012). 
However, some participants described understanding the experience to be more positive and helpful, particularly following debrief:

... They told me how aggressive and unpredictable I was before seclusion. I understood that this was the only alternative and a part of my treatment... (Kontio et al. 2012 p.20).

iv) Suggestions for Improvements

Many participants in the papers were able to reflect on their experiences of seclusion and volunteer suggested improvements. These were for more sensory stimulation in the seclusion room itself and for more interpersonal support from staff:

I reckon they should have paintings on the walls or on the roof or something... I don't know, anything to keep your mind occupied... I think it was worse for me in a way because I was so bored... (Meehan et al., 2000).

Talk about it - talk through the feelings and offer some kind of touching that would be reassuring (Norris and Kennedy 1992 p. 12).

\section{$\underline{\text { Interactions with Staff }}$}

All but one (4) of the papers presented themes exploring the role of staff as an important aspect of the experience of seclusion. Interactions with staff were important themes in both positive and negative interactions. They were also pertinent to participants' perceptions of the provision or absence of support in making sense of the process and experience of seclusion. Both positive and negative aspects of participants' interactions with staff were reported. Staff members attending the seclusion room were either described as 'compassionate' or 'the silent guard' (Wadeson and Carpenter 1976 p. 324). Positive aspects of interactions with 
staff, where clear communication, support and understanding were shown, was described in five papers $(1,3,8,10,11)$, e.g.

For me I remember that I was about to start screaming but that this nurse stroked my hair and I thought that was such a sweet thing to do. I was deeply moved, and then I calmed down completely and the urge to scream was over. Just that little gesture of stroking my hair. Yes I thought it was very sweet (Hoekstra et al. 2004).

In contrast the negative aspects about poor quality interactions and a lack of communication or concern was identified in seven papers:

...the staff is for patients. I did not like it that two nurses stood indifferently near me in the seclusion room and talked by themselves... (Kontio et al. 2012 p. 20).

The role of staff in understanding and knowing the individual may be key to informing the interactions. Participants from one paper (6) described how if staff had better understood them or had known about their backgrounds and treatment plans they would not have been secluded:

I feel that if they'd have known that I was claustrophobic and a little of my background, the outcome could have been different. I mean being cooped up in one floor, you can't really exercise. And that's how I was trying; normally I would blow off steam that away... (Faschingbauer et al 2013).

The overall synthesis of the existing qualitative literature on the lived experience of seclusion for adults with mental health is that seclusion has the potential to cause iatrogenic harm. The role that staff members play in seclusion is key to whether the experience of seclusion is to be harmful or helpful. Clear, open and compassionate interactions and support from staff, including during debrief, were described in the context of more positive experiences. Harmful 
experiences were described in the context of uncompassionate care, inadequate support, information and inattention to basic human needs.

\section{Discussion}

This paper set out to conduct a systematic search of the existing literature on the first-hand experiences of seclusion for adults with mental health difficulties, to appraise the quality of the literature and synthesise findings. A small number of small-scale qualitative studies were found, which were found through systematic appraisal to vary in quality. These explored the experience of seclusion using questionnaires and interviews conducted in Australia, New Zealand, Finland, The Netherlands, Canada and USA and dated from 1976 to 2013. It is acknowledged that cultural and legal differences between countries across time will influence practices in the use of seclusion and therefore possible the experiences of those placed in seclusion.

Seclusion is a controversial treatment used on inpatient mental health units for the short-term management of potentially dangerous behaviour. Guidelines stipulate that it must only be used as a last resort and measures should be taken to ensure that the dignity of service users is upheld throughout the seclusion process. Despite controversy, there is very little scientific research investigating the underlying theoretical principles proposing the use of seclusion.

Guidelines for the use of seclusion in Western countries, such as the UK (NICE, 2005) USA, Australia, and Canada (El-Badri and Mellsop, 2008; Faschingbauer et al. 2013; Larue et al. 2013) stipulate that seclusion should be used as a last resort in the short term management of violent and aggressive behaviour. Experiences recounted by participants suggest that these guidelines may not always be followed in clinical practice. The studies included in this review were conducted across different geographical regions with a wide temporal range 
dating from 1976 to 2013 . A criticism of the JBI review method is that it does not account for potential cultural differences. However, unexpectedly this review found that findings about the experience of seclusion were consistent across the findings from different countries and time periods and did not appear to suggest cultural differences. There may be cause for concern if future research does not find changes in the experience of seclusion to reflect changes in guidelines on the use of seclusion. That is, it would be hoped that future findings would reflect improvements in the overall experience of seclusion, including reductions in perceptions of issues such as dehumanising effects of seclusion, problems with meeting basic needs or experiences or being punished. Equally, however, it is acknowledged being placed in seclusion is unlikely to be experienced by all individuals as therapeutic or beneficial. It was surprising that there were no papers exploring service users' experiences of seclusion from the UK, particularly given that it is in common use and subject to national guidelines (CQC, 2010; NICE, 2005).

It is acknowledged that the included studies only looked at the individual service-users' perceptions of their experiences of seclusion and these were not corroborated with staff reports or records of what happened. Although there is no way of knowing what actually happened during those events described, it was clear from the findings that individuals had been exposed to situations where treatment was experienced as punishing, or where they did not have access to facilities in order to attend to their basic needs, such as going to the toilet, in keeping with the findings of the CQC (2010). Further research is needed to explore why this might be. There could also be scope for action research to implement the improvements suggested by participants in some of the reviewed studies and evaluate the impact of improvements on people's experience of seclusion, or indeed to reduce the use of seclusion through the use of alternative strategies (e.g. Kontio et al., 2010; Taxis, 2002). 
A number of positive experiences were identified, highlighting examples of good practice and the importance of clear and open communication from staff, compassionate care and working to understand the person and to support them in learning from their own behaviour. It is likely that nurses and other staff involved in seclusion are aware of the potential seclusion holds for causing iatrogenic harm. However, it may be helpful to raise their awareness of the evidence that their own individual actions may contribute to or prevent such harm, to influence how seclusion is used, even if there is no reduction in frequency of use. It is acknowledged that awareness in itself may not be sufficient to lead to significant change and it may take a number of years to establish a paradigm shift towards alternatives to seclusion within services, as seen in the example reported by Taxis (2002).

Evidence on the effectiveness of seclusion is lacking, with some reviews reporting that it is an effective intervention for the short-management of aggression (Fisher, 1994; Lendemeijer and Shortridge-Baggett, 1997) and others highlighting the poor quality of such research and potential biases. One of the findings identified in this systematic literature review was the participants described that the seclusion process led them to become submissive and less open with others. This is an important observation that needs to be acknowledged. It raises the question whether the use of seclusion aims to help individuals manage their distress or to coerce them to behave in a more desirable manner. Future research into the 'effectiveness' of seclusion should take a critical approach to how 'effectiveness' is operationalized.

Gutheil (1978) described observations on the theoretical underpinnings of seclusion. He described that seclusion works on the basis of containment, isolation and reduced sensory input, but there has been little development of theory to practice links for seclusion since. Whilst some participants in the current review described as helpful containment and being restricted to a safe place where they were unable to harm themselves or others, many 
described the experiences that contradict the theoretical propositions of seclusion. For example, for many, reduced sensory stimulation had the opposite effect to what would be expected from Gutheil's observations of isolation in seclusion and therapeutic aims of seclusion. Participants from the existing literature described negative effects of sensory stimulation, and described ways of coping such as singing or becoming more agitated to cope with this. These coping behaviours in response to the unusual experience of being placed in seclusion run the risk of being interpreted by staff as pathological. Therefore it is vital that staff members are able to understand how individuals may express and cope with distress. Gutheil's observations on isolation are also contradicted by the finding that participants who described more positive experiences of seclusion often did so in the context of compassionate interactions with staff and knowing that they were not alone. Many described that the experience of feeling alone increased their distress. Further research investigating the positive experiences of seclusion is necessary. It is clear from the synthesis that the role of staff and the quality of their communication, relationship and interactions with the individual is very important.

\section{Limitations}

It is important to consider the limitations of the present review. Its focus on firsthand, qualitative accounts of service users' aimed to bring to light the views and experiences of individuals who have been subjected to the use of seclusion. However, this naturally limits the number of papers included, as quantitative studies were excluded, as were those where it was not possible to distinguish data from service users and staff. As no date limits were set on the systematic search, included papers dated back as far as 1976, although three of the 11 included papers were published in the 2000s and five since 2010. Practice in seclusion will have changed across that time period and will also be different within the cultural and legal frameworks of the six countries in which studies were conducted. As discussed, the 
findings across the papers were surprisingly consistent, but as policy and practice develop it is possible that future research could have different findings.

\section{$\underline{\text { Conclusion }}$}

The overall synthesis of findings concluded that seclusion has the potential to cause iatrogenic harm to adults with mental health difficulties. The role of staff appears to be key to whether the experience is recounted as positive or negative. If this is true, then there are indications for staff to understand and be sensitive to how their interactions may minimise or maximise iatrogenic harm. Some of the papers reviewed indicate that debrief and reflection afterwards with the service-user and compassion from staff throughout are important factors to minimise iatrogenic harm. Further research is needed to identify a theoretical basis and better understanding of those processes that can lead to seclusion being experienced as a helpful or harmful intervention and to build evidence-based policies and guidelines for best practice. 


\section{$\underline{\text { References }}$}

Alty, A. and Mason, T. (1994), Seclusion and Mental Health, Chapman and Hall: London. Antoniou, J. (2007). "Commentary". M. Hardcastle, D. Kennard, S. Grandison, and L. Fagin: Experiences of Mental Health Inpatient Care, East Sussex, UK: Routledge, pp. 124 126.

Ashcraft, L. and Anthony, W. (2008,), "Eliminating seclusion and restraint in recoveryoriented crisis rervices", Psychiatric Services, Vol. 59 No. 10, pp. 1199-1202.

Care Quality Commission (2010). Count me in 2010 London: Author.

Care Quality Commission (2015). Brief guide: seclusion rooms, available at: https://www.cqc.org.uk/sites/default/files/CQC\%20mental\%20health\%20brief\%20guide \%202\%20-\%20seclusion\%20rooms.pdf 
Donat, D. C. (2003), "An analysis of successful efforts to reduce the use of seclusion and restraint at a public psychiatric hospital”, Psychiatric Services, Vol. 54 No. 8, pp. 11191123.

Duxbury, J. and Whittington, R. (2005), "Causes and management of patient aggression and violence: staff and patient perspectives", Journal of Advanced Nursing, Vol. 50 No. 5, pp. 469-478.

El-Badri, S. and Mellsop, G. (2008), "Patient and staff perspectives on the use of seclusion", Australian Psychiatry, Vol. 16 No. 4, pp. 248-252.

Farrell, G. and Dares, G. (1996), "Seclusion or solitary confinement: therapeutic or punitive treatment?", Australian and New Zealand Journal of Mental Health Nursing, Vol. 5 No. 4, pp. 171-179.

Faschingbauer et al. (2013), "Use of seclusion: finding the voice of the patient to influence practice", Journal of Psychosocial Nursing, Vol. 51, No. 7, pp. 32-38.

Fisher, W. A. (1994), "Restraint and seclusion: a review of the literature", American Journal of Psychiatry, Vol. 151 No. 11, pp. 1584-1591.

Frueh et al. (2005), "Pateints' reports of traumatic or harmful experiences within the psychiatric setting", Psychiatric Services, Vol. 56 No. 9, pp. 1123-1133.

Gutheil, T. (1978), "Observations on the theoretical bases for seclusion of the psychiatric inpatient”, American Journal of Psychiatry, Vol. 135 No. 3, pp. 325-328.

Geller, J. L. (2007), "Restraint and seclusion: the model eliminating use in health care", Psychiatric Services, Vol. 58 No. 6, pp. 883-884.

Happell, B. and Harrow, A. (2010), "Nurses' attitudes to the use of seclusion: a review of the literature", International Journal of Mental Health Nursing, Vo. 19 No. 3, pp. 162-168. Hoekstra, T. et al. (2004), "Seclusion: The inside story", Journal of Psychiatric and Mental Health Nursing, Vol. 11 No. 3, pp. 276-283. 
Joanna Briggs Institute (2009), CRSTP Study Guide: The Systematic Review of Evidence Generated by Qualitative Research, Narrative and Text, Australia: The Joanna Briggs Institute.

Khalifeh, A.H. (2015), "Position statement: The use of seclusion in psychiatric settings". Middle East Journal of Nursing, Vol. 9, No. 3., pp. 31 - 36.

Kontio, R. et al. (2010), "Patient restrictions: are there ethical alternatives to seclusion and restraint?", Nursing Ethics, Vol. 17, No. 1, pp. $65-75$.

Kontio, R. et al. (2012), "Seclusion and restraint in psychiatry: patients' experiences and practical suggestions on how to improve practices and use alternatives", Perspectives in Psychiatric Care, Vol. 48 No. 1, pp. 16-24.

Larue, C. et al. (2013), "The experience of seclusion and restraint in psychiatric settings: perspectives of patients", Issues in Mental Health Nursing, Vol. 34, No. 5, pp. 317-324.

Lendemeijer, B. and Shortridge-Baggett, L. (1997), "The use of seclusion in psychiatry: a literature review", Scholarly Inquiry for Nursing Practice, Vol. 11, No. 4, pp. 299-320. Martin, A. et al. (2008), "Reduction of restraint and seclusion through collaborative problem solving: a five-year prospective inpatient study", Psychiatric Services, Vol. 59 No. 12, pp. 1406-1412.

Mason, T. (1993), "Seclusion theory reviewed - a benevolent or malevolent intervention?", Medicine, Science and Law, Vo. 33 No. 2, pp. 95-102.

Mayers, P., Keet, N., Winkler, G. and Flisher, A.J. (2015). "Mental health service users' perceptions and experiences of sedation, seclusion and restraint", International Journal of Social Psychiatry, Vol. 56, No. 1, pp. $60-73$.

McCoy, S. M. and Garritson, S. H. (1983), "Seclusion: the process of intervening", Journal of Psychosocial Nursing and Mental Health Services, Vol. 21 No. 8, pp. 8-15. 
Meehan, T. et al. (2000), "Patients' perceptions of seclusion: a qualitative investigation", Journal of Advanced Nursing, Vol. 31 No. 2, pp. 370-377.

Moran, A. et al. (2009), "Restraint and seclusion: a distressing treatment option?" Journal of Psychiatric and Mental Health Nursing, Vol. 16 No. 7, pp. 599-605.

Muir-Cochrane, E. (1996), "An investigation into nurses' perceptions of secluding patients on closed psychiatric wards", Journal of Advanced Nursing, Vol. 23, No. 3, pp. 555-563

Nelstrop, L. et al. (2006), "A systematic review of the safety and effectiveness of restraint and seclusion as interventions for the short-term management of violence in adult psychiatric inpatient settings and emergency departments", Worldviews on EvidenceBased Nursing, Vol. 3 No. 1, pp. 8-18.

NICE (2015). Violence and Aggression: Short-Term Management in Mental Health, Health and Community Settings. UK: NICE

NICE (2005). Violence: The Short-Term Management of Disturbed/ Violent Behaviour in InPatient Psychiatric Settings and Emergency Departments. UK: NICE

Norris, M. K. and Kennedy, C. W. (1992), "The view from within: how patients perceive the seclusion process", Journal of Psychosocial Nursing, Vol. 30 No. 3, pp. 7-13.

O'Brien, L. and Cole, R. (2004). "Mental health nursing practice in acute psychiatric closeobervation areas". International Journal of Mental Health Nursing, 13, pp. 89 - 99.

Richardson, B. K. (1987), "Psychiatric inpatients' perceptions of the seclusion-room experience", Nursing Research, Vol. 35 No. 4, pp. 234-238.

Rosen, H. and DiGiacomo, J. (1978), "The role of physical restraint in the treatment of psychiatric illness", Journal of Clinical Psychiatry, Vol. 39 No. 3., pp. 228-232. 
Sailas, E. E. S. and Fenton, M. (2012), "Seclusion and restraint for people with serious mental illnesses", The Cochrane Database of Systematic Reviews (issue 6). DOI: 10.1002/14651858.CD001163.

Sambrano, R. and Cox, L. (2013), "I sang Amazing Grace for about 3 hours that day': Understanding Indigenous Australians' experience of seclusion”, International Journal of Mental Health Nursing, Vol. 22 No. 6, pp. 522-531.

Taxis, J.C. (2002). "Ethics and praxis: alternative strategies to physical restraint and seclusion in a psychiatric setting". Issues in Mental Health Nursing, Vol. 23, No. 2, pp. $157-170$.

Wadeson, H. and Carpenter, W. T. (1976), "Impact of the seclusion room experience", The Journal of Nervous and Mental Disease, Vol. 163 No. 5, pp. 318-328.

Wells, A. (1972), "The use of seclusion on aa university hospital psychiatric floor", Archives of General Psychiatry, Vol. 26 No. 5, pp. 410-413. 